\title{
Presencia de partidos políticos y diputados en Internet en Argentina, Paraguay y Uruguay
}

\author{
Yanina Welp, ${ }^{*}$ Alejandra Marzuca**
}

\author{
Perfiles Latinoamericanos, 24(47) \\ 2016 | pp. 199-224 \\ DOI: $10.18504 / \mathrm{pl} 2447-011-2016$
}

\begin{abstract}
Resumen
Según Latinobarómetro, los partidos políticos son las instituciones en las que menos confían los latinoamericanos. Esto no es particular de América Latina, sino una tendencia en las democracias occidentales. En este contexto, en Europa y Estados Unidos, entre otros, los medios digitales han pasado a ser un instrumento para superar la crisis de la representación, por su potencial para renovar la política, y porque permiten una mayor transparencia y la apertura de nuevos canales de contacto y comunicación entre ciudadanos y representantes. ¿En qué medida y con qué particularidades dicha adopción sucede en América Latina? A partir del estudio de la presencia en línea de los partidos políticos y los diputados de Argentina, Paraguay y Uruguay (webs, blogs, Facebook y Twitter), este artículo explora los niveles de adopción de medios digitales y la influencia de variables como la difusión de Internet en el país, la crisis de representación y las características de los partidos políticos (institucionalización, ideología, tamańo, papel promotor de figuras destacadas) como condicionante del alcance y los rasgos de esta adopción.
\end{abstract}

\begin{abstract}
According to Latinobarómetro, political parties are the institutions which less confidence receive from Latin Americans. This is not a particularity of Latin America, but a documented problem of Western consolidated democracies. In this context, in Europe and United States, among others, the use of digital media become a tool to overcome the crisis of representation, given their capacities to renovate politics, and because they allow the offering of more publicity of public matters as well as the opening up of new channels of participation and communication between citizens and representatives. But to what extent and with which features is this adoption happening in Latin America? Based on the study of the online presence of political parties and deputies of Argentina, Paraguay and Uruguay (webs, blogs, Facebook and Twitter), this article explores the levels of digital media adoption and the influence of variables such as the internet diffusion in the country, the crisis of representation and the characteristics of political parties (institutionalization, ideology, size, role of leaders) explaining the extension and characteristics of this adoption.
\end{abstract}

Palabras clave: medios digitales, e-democracia, redes sociales, partidos políticos, representantes, TIC. Keywords: digital media, e-democracy, social networks, political parties, parliamentarians, ICTs.

* Centre for Research on Direct Democracy (C2D).

** IDEL (Universidad Católica del Uruguay). 


\section{Introducción ${ }^{1}$}

L

últimas décadas han visto el crecimiento de los estudios dedicados a evaluar los alcances y consecuencias políticas de la entrada de las viejas democracias occidentales en la era de la información. Por el contrario, son escasas las investigaciones que abordan la cuestión en Latinoamérica. ${ }^{2}$ Una de las razones de esa falta de interés puede asociarse a la brecha digital en la región. Algunos investigadores consideran que los efectos políticos de Internet sólo pueden ser analizados si la red es ampliamente utilizada en la sociedad de referencia. Por ejemplo, Setälä \& Grönlund (2006: 153) sólo estudian países con más de un $70 \%$ de usuarios. Asumir dicha premisa excluiría todos los casos sudamericanos, para los que la media de usuarios es del $46 \%$ (cálculo propio basado en datos de ITU 2012 para diez países de América del Sur). Aquí rechazamos este supuesto porque entendemos que Internet y las redes sociales tienen una influencia directa e indirecta sobre las sociedades (Anduiza, Jensen \& Jorba, 2012). Asimismo, todos los países latinoamericanos han implementado políticas orientadas a la introducción de la tecnología en los distintos ámbitos del hacer social (Welp, 2008; Breuer \& Welp, 2014). El número de internautas crece paulatinamente en toda América Latina mientras sus parlamentos, partidos y representantes han comenzado a utilizar estos espacios virtuales para presentar información y comunicarse con la ciudadanía, cuestión que replican los gobiernos con sus diferentes direcciones y unidades administrativas (Hunter, 2011).

Pero ¿̇en qué medida y con qué consecuencias se adoptan estos medios digi$\operatorname{tales}^{3}$ en el terreno político latinoamericano? Este trabajo se propone contribuir al análisis del estado de la cuestión centrándose en el estudio de los partidos

1 Los primeros resultados de esta investigación fueron presentados en el xxx congreso de la Latin American Studies Asociation (San Francisco, 23-26 mayo de 2012) y en el iv Congreso Uruguayo de Ciencia Política (14-16 de noviembre de 2012). Agradecemos a Anita Breuer y a los participantes en estos eventos por los comentarios recibidos. Una versión que incluye el análisis de webs parlamentarias fue publicada en Welp \& Marzuca (2014).

2 Estos estudios se centran en el análisis de las webs parlamentarias y/o en la difusión del gobierno electrónico y en el voto electrónico. Véase, por ejemplo, Frick (2005), López et al. (2008), Araya \& Barría (2009), Welp $(2008,2011)$ y Loza (2011). Recientemente se han incrementado los estudios centrados en los usos de medios digitales por parte de la sociedad civil (Breuer \& Groshek, 2014; Burch \& León, 2014). Sobre transparencia Fumega \& Scrollini (2012); o sobre el uso de sistemas de orientación del voto Marzuca et al. (2011) y Twitter en elecciones (Llorente \& Cuenca 2014).

3 No existe una definición unificada de medios digitales. Aquí usamos el concepto como parte del paraguas más general de las tecnologías de la información y las comunicaciones (TIC) para referirnos a los medios de comunicación basados en Internet, que permiten la difusión e intercambio de información y la formación de redes sociales virtuales. 
políticos con representación en la Cámara Baja y los diputados de Argentina, Paraguay y Uruguay. Son varios los objetivos: $i$ ) analizar el alcance de la adopción de medios digitales (webs, blogs, Facebook y Twitter) por parte de partidos políticos y representantes de los países mencionados; ii) analizar las variables que podrían condicionar una mayor o menor adopción (contextuales y estructurales), y iii) con base en lo anterior, reflexionar sobre las estrategias comunicacionales desplegadas en la red.

Para cumplir con lo planteado se presenta una revisión de la literatura sobre el tema; luego se describe la metodología (hipótesis, criterios de selección de casos y propuesta analítica); enseguida se analiza la oferta de información y espacios de interacción de los partidos con representación en la Cámara Baja, la presencia y usos que los diputados de los tres países hacen de las webs y las redes sociales Facebook y Twitter, y la actividad de los diputados según partido político. Finalmente se presentan las conclusiones.

\section{Estado del arte}

¿Por qué y para qué los partidos políticos y representantes usarían los medios digitales? Según la encuesta del Latinobarómetro (1996-2011), los partidos políticos son sistemáticamente las instituciones en las que menos confían los latinoamericanos. Los parlamentos, aunque un poco mejor ubicados, también se encuentran entre los actores desacreditados entre la población. Sin embargo, la desconfianza en las instituciones de la democracia representativa no es privativa de la región sino un problema compartido con las democracias de países desarrollados (Dalton \& Weldon, 2007). Algunos autores consideran que los partidos políticos viven una fase de transición, que han visto disminuir sus niveles de apoyo mientras la acción política se manifiesta a través de nuevas organizaciones y movimientos sociales (Norris, 2001; Lusoli et al., 2005). En este contexto, la difusión vía los medios digitales se ha identificado como una potencial herramienta para superar la mencionada crisis, ya que permite el incremento de la información a disposición del público, y permite abrir canales para la participación ciudadana en la definición de políticas públicas (Borge, 2005; Setälä \& Grönlund, 2006; Dahl, 1989). Se ha subrayado además que son medios que permiten establecer más y mejores lazos entre representantes y representados, fortalecidos por nuevas formas de interacción (Padró-Solanet \& Cardenal, 2008; Borge, 2005; Hague \& Loader, 1999; Lusoli et al., 2005).

Más allá de las promesas de cambio o transformación, la investigación con base empírica muestra que algunas instituciones — los partidos, en especialhan sido más limitadas y conservadoras en su adopción de tecnología que los 
ciudadanos y organizaciones de la sociedad civil (Borge, 2005; Trechsel et al., 2003; Castells \& Sey, 2004). Los estudios de casos europeos, Australia y América del Norte, señalan que ha habido una rápida adopción de la Web por parte de los partidos, mientras que los usos y consecuencias de la misma, son menos claros. La rápida adopción parece condicionada por el crecimiento de Internet (y éste, por la riqueza del país), mientras la intensidad y estrategias específicas podrían estar condicionadas por cuestiones como el tamaño y tipo de partido, sus objetivos, su posición en el mercado electoral y su relación con los medios de comunicación (Padró-Solanet \& Cardenal , 2008; Rommël, 2003). Las conclusiones más corrientes sugieren que en general los partidos utilizan sus webs para publicar información básica, a la vez que mantienen estrategias comunicacionales tradicionales y procesos jerárquicos en la toma de decisiones (Nixon \& Johansson, 1999; Kamarck, 1999; Norris, 2001).

En cuanto a la ideología, algunos trabajos (Hartleb, 2013; De Rosa, 2013; Welp \& Wheatley, 2009) han enfatizado en los exitosos usos de las redes sociales de partidos extremistas que no encuentran espacio en los medios tradicionales. Esto se asocia con la potencialidad ofrecida por los medios digitales para alterar la estructura de producción y circulación de información, ampliando los canales existentes y difuminando las barreras entre la producción y el consumo de información (Howard, 2005). Estas barreras son evidentes en medios masivos de comunicación como la prensa escrita y la televisión tradicionales, en que los roles de productores y consumidores están claramente diferenciados y hay filtros de entrada que reducen la llegada de, por ejemplo, partidos radicales o antisistema, hasta que su éxito en los medios digitales fuerza la apertura de los medios tradicionales (Welp \& Wheatley, 2009). Por último, el contexto también influye fuertemente estimulando la adopción de tecnología según la comunicación política se presente como un fenómeno multilateral de intervenciones combinadas de webs de partidos, comunidades virtuales, redes de blogs, marketing viral y otras manifestaciones retroalimentadas con los medios periodísticos y audiovisuales convencionales (Dader, 2009: p. 52).

Asimismo, hay estudios que se enfocan en la presencia y las estrategias que, en lo individual, los parlamentarios (representantes y senadores) tienen en las redes, considerando variables similares a los casos de análisis de la presencia web de los partidos (ideología, por ejemplo), a lo que suman género, edad y adscripción partidaria (Sæbø, 2011). Sin embargo, esa investigación no incluye los casos latinoamericanos, ${ }^{4}$ lo que constituye una de las contribuciones de este trabajo.

4 Una excepción es Norris (2001); no obstante, el análisis de esta autora se circunscribe a la web de los partidos y al análisis de la relación entre la riqueza de un país y el despliegue de webs partidarias. 


\section{Metodología}

\section{Hipótesis}

De la revisión de la literatura se desprenden algunas hipótesis exploratorias que guían este artículo. Mismas que combinan dos tipos de condiciones que influyen tanto en la adopción como en las estrategias que partidos y representantes desarrollan en los medios digitales: condiciones contextuales y condiciones estructurales. Las primeras consideran el contexto particular en el que partidos y representantes se encuentran inscritos, refieren a la influencia que podría tener la crisis de representación y el alcance de la difusión de Internet impulsando o ralentizando la adopción de tecnología (Calderaro, 2014; Norris, 2001; Trechsel et al., 2003). Las segundas son las condiciones estructurales que permitirían diferenciar estrategias al interior de un sistema de partidos, y que refieren a características de los partidos (Rommël, 2003; Gibson \& Ward, 2000; PadróSolanet \& Cardena, 2008; López, 2005). Definimos cuatro conjuntos de hipótesis asociadas a este esquema:

a) Crisis de representación: contextos en que se observe una menor confianza en los partidos, podrían incentivar a que éstos incorporen un uso novedoso de los medios digitales. Al ofrecer más información y mayores canales de participación por esta vía, los partidos buscarían mantener el interés de afiliados y adherentes y atraer nuevos miembros (incentivo contextual). Sin embargo, este contexto combinado con una baja institucionalización de los partidos podría ofrecer incentivos individuales a ganar apoyos por fuera o por encima de las organizaciones partidarias, fomentando el personalismo.

b) Difusión de Internet: a mayor difusión de Internet en un país, mayores serán los incentivos para que partidos y representantes adopten y usen con intensidad los medios digitales. Esto, asumiendo que la sociedad de referencia cuenta con una masa crítica que utiliza esos medios retroalimentando la participación de otros actores, entre los que destacan los medios masivos de comunicación y los políticos (incentivo contextual).

c) El grado de institucionalización del sistema de partidos ${ }^{5}$ podría influir en el uso que los partidos hacen de la Web y redes sociales, esto es, limitado a la oferta de información (tradicional) o abierto a la participación (innovador). Sistemas altamente institucionalizados favorecen usos tradicionales, mientras que los de baja institucionalización realizan usos más innovadores. En el primer caso, se

5 Considerando las dimensiones que proponen Mainwaring \& Torcal (2005): volatilidad electoral, arraigo de los partidos en la sociedad, legitimidad de los partidos y la fortaleza de la organización partidaria.

Y. Welp, A. Marzuca | Presencia de partidos políticos y diputados en Internet en Argentina, Paraguay y Uruguay Perfiles Latinoamericanos, 24(47) | FLACso México | pp. 199-224 | DoI: 10.18504/pl2447-011-2016 
esperaría que partidos pequeños y nuevos, con menos espacio en los medios clásicos (prensa escrita, televisión y radio) sean comparativamente más activos en los medios digitales. Mientras que el segundo escenario, de organizaciones partidarias débiles y liderazgos individuales fuertes, favorece la presencia individual de los referentes políticos en las redes sociales, sin encontrar un correlato de presencia en línea sólida (ausencia de web de partido) o encontrando ejemplos de poco desarrollo y escasa o nula innovación (incentivo estructural).

d) "Efecto contagio": el uso político de redes sociales incluye a miembros del Poder Ejecutivo, senadores y otros referentes del ámbito nacional. Suponemos que los partidos con líderes convencidos e involucrados en la utilización de redes sociales tienen más motivos para incorporar estas herramientas a escala interna, en contraste con los casos donde no existe esta proactividad (incentivo estructural).

Finalmente, exploraremos qué tanto las variables individuales (género, edad, nivel de educación, liderazgo) condicionan la presencia y actividad en línea de los parlamentarios y cuánto influyen las características de los medios sobre su difusión en la actividad política. Por ejemplo, la dinámica del perfil de Facebook es restringida en tanto limita el número de contactos, mientras una página web amplía el público potencial, aunque privilegia las formas de comunicación tradicional (difusión de información). O bien, Twitter da pauta a una comunicación más abierta a la vez que facilita el seguir a muchos usuarios (Grant et al., 2010).

\section{Criterios de selección de los casos}

En este artículo se analiza a tres países que muestran diferencias relacionadas con las hipótesis arriba planteadas. En cuanto a la confianza de los ciudadanos en sus parlamentos y partidos $(\mathrm{Ha})$, según datos del Latinobarómetro, Paraguay y Argentina presentan muy bajos niveles, mientras que son mejores los resultados en Uruguay, si bien no tan alentadores.

La diferencia en el patrón de difusión de Internet $(\mathrm{Hb})$ también se incorporó en este análisis. Al iniciar la investigación, con base en las otras variables mencionadas y en datos de ITU 2009, se optó por Uruguay (42\%, difusión alta en términos relativos), Argentina (34\%, difusión intermedia) y Paraguay (19\%, difusión baja). Sin embargo, al volver a estos datos — una vez finalizado el trabajo empírico - se encontró un cambio destacado que mostraba la velocidad de crecimiento de la red en los países sudamericanos y alteraba nuestra clasificación, ya que, mientras Paraguay se mantenía en el último sitio, Argentina ocupaba ya el segundo lugar (después de Chile) superando a Uruguay (57 y $54 \%$, respectivamente) (tabla 1). Al contrastar el indicador de desarrollo huma- 
no (IDH) o la renta per cápita de los países y sus niveles de difusión de Internet, se observa la correlación existente. ${ }^{6}$

Tabla 1. Indicadores de confianza en parlamentos y partidos, usuarios de Internet, desarrollo humano y renta per cápita en los países de América del Sur (2010-2011)

\begin{tabular}{|c|c|c|c|c|c|c|}
\hline \multirow[t]{2}{*}{ País } & \multicolumn{2}{|c|}{ Confianza $^{1}$} & \multicolumn{2}{|c|}{ Usuarios de Internet $\%^{2}$} & \multirow[t]{2}{*}{$I D H^{3}$} & \multirow[t]{2}{*}{ Renta per cápita } \\
\hline & Parlamento & Partidos políticos & 2009 & 2012 & & \\
\hline Argentina & 13 & 10 & 34 & 56.8 & $0.797(\mathrm{MA})$ & 18319 \\
\hline Bolivia & 27 & 8 & 16.8 & 35.5 & $0.663(\mathrm{M})$ & 4995 \\
\hline Brasil & 23 & 13 & 39 & 48.5 & $0.718(\mathrm{~A})$ & 12181 \\
\hline Chile & 11 & 5 & 41.5 & 61 & $0.805(\mathrm{MA})$ & 17974 \\
\hline Colombia & 29 & 20 & 30 & 49 & $0.710(\mathrm{~A})$ & 10742 \\
\hline Ecuador & 5 & 4 & 24.6 & 35 & $0.720(\mathrm{~A})$ & 8854 \\
\hline Paraguay & 9 & 5 & 18.9 & 29 & $0.665(\mathrm{M})$ & 5294 \\
\hline Perú & 7 & 4 & 31 & 38 & $0.725(\mathrm{~A})$ & 10589 \\
\hline Uruguay & 55 & 40 & 41.8 & 54.5 & $0.783(\mathrm{~A})$ & 15786 \\
\hline Venezuela & 42 & 15 & 32.7 & 49 & $0.735(\mathrm{~A})$ & 13070 \\
\hline
\end{tabular}

Fuente: Elaboración propia basada en las fuentes que se listan a continuación.

${ }^{1}$ Latinobarómetro. Informe 2011. Se pedía a los encuestados el señalamiento de "cuánta confianza tiene usted en" cada institución indicada (mucha, algo, poca o ninguna). En la tabla se registra la suma de "mucha" y "algo".

2 International Telecommunication Union 2010 (El indicador registra el uso de Internet, desde cualquier lugar, en los últimos doce meses por las personas incluidas en el estudio).

3 Índice de Desarrollo Humano. PNUD 2011. (MA: muy alto; A: alto; M: medio; B: bajo).

${ }^{4}$ FMI. Base de datos World Economic Outlook. 2012.

La relación entre la cantidad de usuarios de Facebook y el total de población es similar en Argentina y Uruguay (llega casi a la mitad), mientras que en Paraguay se ubica por debajo del 20\% (Socialbakers, 2012). ${ }^{7}$ Las estimaciones de Twitter señalan que, a principios de 2012, Argentina se encontraba entre los veinte países más twitteros del mundo, con más de ochocientas mil cuentas. Había más de medio millón de cuentas vinculadas a Uruguay (datos de 2011) y cerca de ochenta mil en Paraguay (en 2012). ${ }^{8}$ Lo que interesa destacar aquí es la enorme difusión de estas redes sociales y su sostenida tasa de crecimiento. Estos

6 La literatura enfatiza en este vínculo, pero señala que no hay relación lineal entre la riqueza, las características y alcance de la difusión de Internet en distintos países (Hargittai, 1999). Sin embargo, en los casos seleccionados la relación es relativamente lineal.

7 Estas estadísticas revelan un problema que enfrenta la investigación debido a la dificultad para identificar fuentes confiables, lo que se debe tanto a la dinámica de los medios estudiados como a las diversas formas de medir su expansión. En este caso, se observa que las estadísticas de penetración de Internet de Socialbakers no coinciden con las ofrecidas por ITU (con una diferencia de más de diez puntos porcentuales en el caso argentino. Confróntese con la tabla 1).

8 Véanse http://www.enter.co/Internet/por-fin-luces-sobre-numero-de-usuarios-de-twitter-en-latinoamerica/ y http://www.paraguay.com/columnas/twitter-el-sexto-sentido-social-69855

Y. Welp, A. Marzuca | Presencia de partidos políticos y diputados en Internet en Argentina, Paraguay y Uruguay Perfiles Latinoamericanos, 24(47) | FLACso México | pp. 199-224 | DoI: 10.18504/pl2447-011-2016 
hechos no conducen a afirmar que modificarán las formas de hacer política, pero sí justifican el interés por realizar investigación del tema.

Los tres países en estudio muestran considerables diferencias en el grado de institucionalización de su sistema de partidos (Hc). A mediados de los noventa, Mainwaring \& Scully (1995) diferenciaban entre los sistemas institucionalizados (Uruguay como ejemplo destacado), sistemas incipientes o no institucionalizados (Argentina como caso controvertido) y sistemas hegemónicos en transición (Paraguay como caso emblemático, junto a México). A pesar del tiempo transcurrido y de haberse registrado numerosas transformaciones, las diferencias entre los tres sistemas aún son considerables: un sistema de partidos muy institucionalizado y estable en Uruguay, uno en crisis en Argentina, y uno también en crisis en Paraguay, pero con un fuerte dominio del Partido Colorado.

Por último, (d) los partidos difieren al interior de cada país en su tamaño, longevidad y acceso a medios tradicionales. En tanto que la publicación directa en las redes sociales funciona para anunciar actividades y opiniones, lo que las constituye en un ámbito del que los medios (radio, televisión y prensa escrita) obtienen y redirigen información. Ejemplos de esto son los segmentos de los portales de prensa dedicados al seguimiento de las cuentas de Twitter de los miembros del parlamento o de personalidades de la política nacional e internacional. ${ }^{9}$ Un ejemplo al respecto es, del Frente para la Victoria, la presidenta argentina Cristina Fernández, cuya cuenta (@CFKArgentina) está vigente desde 2010 y tenía casi tres millones de seguidores a mediados de 2012. En Uruguay, un pionero es el senador Pedro Bordaberry del Partido Colorado, con más de 57 mil seguidores es el político con mayor cantidad de éstos en el país (@pedrobordaberry). Mientras que, en Paraguay, el expresidente Fernando Lugo (@ Fernando_Lugo) alcanzó más de 24 mil seguidores para ser también el político más popular del país en Twitter. ${ }^{10}$

\section{Marco analítico ${ }^{11}$}

\section{Webs de los partidos}

En cuanto a este rubro, en primer lugar se analizó si los partidos seleccionados (aquellos con representación en la Cámara Baja durante el periodo de análisis,

9 políticos@twitter del portal del Espectador.com en Uruguay, las listas de autoridades y políticos en @ abcdigital de Paraguay, y presidentes y políticos de @lanacioncom en Argentina, y las cuentas de Twitter de los propios medios de prensa.

10 Los datos de las cuentas se actualizaron a julio de 2014. Aunque las referencias mantienen su validez desde 2012.

11 La recolección de datos se realizó durante marzo-mayo de 2012. 
2011-2012) contaban con una web propia. Para identificarlas se consideró la concordancia en la denominación del partido, referencia a oficialidad de la página y, cuando apareció más de una página asociada a un partido, indicadores de actualización (agenda de actividades o antigüedad de noticias, por ejemplo).

Cuando se identificó una web, se analizaron sus atributos relativos a la difusión de información, usos novedosos de la tecnología y canales de interacción y participación. Siguiendo a Gibson \& Ward (2000), el estudio de la presencia de los partidos políticos en la Web supone establecer un esquema que, por intermedio de la observación del diseño y contenido de sus páginas, releve el tipo de uso que le asignan los partidos u organizaciones políticas. Las dimensiones de análisis fueron: a) provisión de información: historia del partido o movimiento, programa y estructura en la que se organiza; b) campañalpromoción: eventos y horarios de las reuniones/encuentros políticos, videos y audios de los actos del partido y de las actividades de sus representantes; c) captación de recursos: que el partido habilite vía web la posibilidad de recaudar fondos y reclutar militantes (membresía en línea); d) interacción: promoción de la comunicación bidireccional entre el partido y los ciudadanos, provisión de correos electrónicos de los miembros del partidos o espacios para dejar comentarios; e) promoción de la participación multidireccional: foros de discusión, ligas a redes sociales. Cada uno de estos indicadores se registra como existente o no existente.

Mientras los puntos $a-c$ dan pauta para diferenciar en una escala desde usos más tradicionales (provisión de información) a los más novedosos (campaña y captación de recursos), los puntos $d$ y $e$ permiten distinguir, en una escala, entre usos orientados a la comunicación bidireccional de aquéllos orientados a la participación y deliberación.

\section{Los parlamentarios en línea}

Las webs o blogs personales son un medio de comunicación para publicar notas, comentarios, proyectos de ley, agenda de actividades, fotos o videos. Potencialmente, de esta forma los parlamentarios superan las barreras impuestas por los medios masivos y consiguen así un espacio propio de contacto con la ciudadanía. A la vez, la forma de comunicación es más convencional (el político publica, el ciudadano sólo en algunos casos puede comentar), pues difundir la página o blog requiere de mayor esfuerzo que las redes sociales.

Para el análisis de redes sociales, cabe decir que si un diputado o diputada tiene la presencia en ellas no necesariamente implica que las adopte para su actividad política. La separación que las figuras políticas deben hacer entre vida pública y privada es un tema complejo. Muchos políticos realizan un uso 
público de ciertos aspectos de su vida privada (preferencias deportivas o musicales, acontecimientos familiares, vinculación con su país, barrio o ciudad), o ésta se encuentra cooptada por su rol político. Aunque esta división es discutible, el contenido y la modalidad en la que el o la representante se posicionen en las redes sociales da pautas sobre el uso que les asigna. Así, habrá un uso político cuando:

1) En el nombre o descripción del usuario aparecen referencias a su actividad política: "Presidente de la Cámara de Diputados del Paraguay. Líder de AVANZAR REPUBLICANO (ANR). Ingeniero de profesión y político por pasión. Club Guaranî” (@victorbogadopy); “Diputado nacional electo por la provincia de Buenos Aires y presidente del bloque Nuevo Encuentro” (@sabbatella); “Diputada por el Partido Nacional Presidenta de la Comisión Departamental de Montevideo. Trabajé como funcionaria de la огт. Fui Ministra de Trabajo 1995-99". ${ }^{12}$

2) Presenta un formato visual que permite la plena identificación del usuario con la actividad política: fotografía de perfil en el marco de su actividad política (en el parlamento, el despacho), en algún lugar emblemático (el edificio legislativo, monumentos o sitios del territorio de fácil identificación); acompañado de la bandera del partido o el país, junto con el líder del partido o movimiento u otras figuras políticas relevantes; haciendo uso de los colores, símbolos, números o frases de su colectividad.

3) La información que allí comparte se vincula a su actividad política, incluyendo: agenda de actividades, opiniones sobre temas de actualidad, llamado al debate con otros políticos o ciudadanos, promoción de proyectos de ley, publicación de fotografías o videos en que aparece con otras figuras políticas participando de actividades públicas (de gobierno o en el marco de su investidura), así como en encuentros políticos, acompañado de distintivos partidarios.

Para los objetivos de este artículo, cabe considerar que se observan dos modalidades de presencia en Facebook: el perfil de usuario y la página. El primero establece redes de contacto directo, mientras que las páginas son espacios vinculados a organizaciones o personalidades y en ellas la proximidad de los "amigos" se sustituye por la de los seguidores o suscriptores. Tanto perfiles como páginas permiten que el usuario o administrador publique en su muro virtual frases o comentarios, y comparta ligas multimedia y fotografías. Estas publicaciones pueden ser comentadas por amigos o seguidores. Tales modalidades difieren respec-

12 En su perfil de Facebook: https://www.facebook.com/ana.lia.pineyrua?sk=wall 
to a la circulación de la información, ya que cuando dos perfiles de Facebook son amigos es posible que ambos se mantengan informados de sus respectivas publicaciones, mientras que, en las páginas, la comunicación es unidireccional (sólo los suscriptores reciben información sobre las actualizaciones de la página, aunque sí pueden publicar en el muro de la página). La páginas no tienen límite para los seguidores (que se convierten en tales al activar la función "me gusta”), en tanto que los perfiles individuales tienen su tope en los cinco mil amigos (volveremos sobre este punto).

\section{Análisis}

\section{Las webs de los partidos}

El estudio analizó un total de 41 organizaciones políticas, de las cuales 22 tenían sitios web (53.6\%) (tabla 2). Aunque la imagen inicial sería la de un panorama heterogéneo, la mayoría de los partidos realizan un pobre y marginal uso de las oportunidades que ofrece Internet. Del total de webs analizadas, sólo cuatro ítems son ofertados por más de la mitad de las páginas: el programa de la organización (72.7\%), videos y audios (59\%), espacios para comentar en la Web y ligas a redes sociales (72.2 y $81.8 \%$, respectivamente). Los usos de la Web para reclutar voluntarios, afiliación en línea o recibir donaciones, son los menos extendidos en todos los casos. Ni la actividad de los parlamentarios, ni los correos electrónicos de los miembros del partido (a excepción de que algunos ofrecen la dirección del presidente/secretario del partido o un contacto administrativo), ni los foros, tienen mayor difusión en las webs partidarias (ninguno ofrece foros de debate). Aunque el $81.8 \%$ de las páginas redirigen hacia cuentas del partido en Facebook y Twitter.

Mientras los incentivos sistémicos no parecen jugar un papel para explicar una mayor o menor adopción de webs por parte de los partidos (Uruguay y Paraguay muestran un panorama más desarrollado que el argentino), nuestra tercera hipótesis se valida parcialmente: sistemas de partidos más longevos e institucionalizados han avanzado más en la adopción de páginas webs. Así, en tanto en Uruguay todos cuentan con página web, ${ }^{13}$ en Paraguay esto se reduce al $83 \%$ y en Argentina a $42 \%$ (para detalles de los partidos analizados, véase la tabla 6). En la misma línea, todas las webs uruguayas estudiadas y casi todas las paraguayas ofrecen el programa o manifiesto de principios del partido, mientras

13 El estudio aún deberá complementarse con el análisis de la presencia en la Web de las fracciones que conforman estos partidos. 
esto lo hacen sólo el $61.5 \%$ de las argentinas. Las actividades de los parlamentarios son publicadas por bastante más de la mitad de los partidos argentinos consultados, y por un sólo partido en Paraguay y Uruguay.

Por último, la adopción de tecnología por parte de los partidos muestra un uso más bien limitado a la difusión de información. El análisis, asimismo, muestra más recursos innovadores entre partidos pequeños que entre los grandes: Generación para un Encuentro Nacional (GEN) de Argentina (2\% de bancas) invita a ser un "cibervoluntari@” y a crear una campaña personalizada para la que proveen recursos gráficos oficiales. ${ }^{14}$ El Partido Independiente uruguayo (2\%) y Nuevo Encuentro (2\%) y Unión Cívica Radical de Argentina (16\%) son los únicos que reciben donaciones en línea. La posibilidad de afiliarse vía web se concentra sobre todo en Argentina; ${ }^{15}$ el paraguayo Partido Patria Querida sería un caso similar (también permite registrarse como voluntario).

Tabla 2. Características de los sitios webs de los partidos políticos por país

\begin{tabular}{|c|c|c|c|c|c|c|c|c|}
\hline & \multicolumn{2}{|c|}{ Argentina } & \multicolumn{2}{|c|}{ Paraguay } & \multicolumn{2}{|c|}{ Uruguay } & \multicolumn{2}{|c|}{ Total } \\
\hline & $n^{\circ}$ & $\%$ & $n^{\circ}$ & $\%$ & $n^{\circ}$ & $\%$ & $n^{\circ}$ & $\%$ \\
\hline Cantidad de organizaciones políticas (OP) & 31 & 100 & 6 & 100 & 4 & 100 & 41 & 100 \\
\hline OP con sitio web & 13 & 41.9 & 5 & 83.3 & 4 & 100 & 22 & 53.6 \\
\hline \multicolumn{9}{|l|}{ Información disponible } \\
\hline Historia del partido & 3 & 23 & 4 & 80 & 2 & 50 & 9 & 40.9 \\
\hline Programa, manifiesto, declaración de principios & 8 & 61.5 & 4 & 100 & 4 & 100 & 16 & 72.7 \\
\hline Estructura del partido y reglamentación & 5 & 38.4 & 3 & 60 & 2 & 50 & 10 & 45.4 \\
\hline \multicolumn{9}{|l|}{ Campaña } \\
\hline Agenda & 6 & 46.1 & 2 & 40 & 4 & 100 & 12 & 54.5 \\
\hline Videos y/o audios & 6 & 46.1 & 4 & 80 & 3 & 75 & 13 & 59 \\
\hline Actividades de sus parlamentarios & 9 & 69.2 & 1 & 20 & 1 & 25 & 11 & 50 \\
\hline \multicolumn{9}{|l|}{ Generación de recursos } \\
\hline Unirse al partido en línea & 5 & 38.4 & 1 & 20 & 0 & 0 & 6 & 27.2 \\
\hline Donar dinero & 2 & 15.3 & 0 & 0 & 1 & 25 & 3 & 13.6 \\
\hline Reclutamiento de voluntarios & 1 & 7.6 & 1 & 20 & 0 & 0 & 2 & 9 \\
\hline \multicolumn{9}{|l|}{ Interacción } \\
\hline Correos electrónicos de los miembros del partido & 2 & 15.3 & 1 & 20 & 2 & 50 & 5 & 22.7 \\
\hline Espacio para comentar el sitio web & 8 & 61.5 & 5 & 100 & 3 & 75 & 16 & 72.7 \\
\hline \multicolumn{9}{|l|}{ Promoción de la participación } \\
\hline Foros en línea & 0 & 0 & 0 & 0 & 0 & 0 & 0 & 0 \\
\hline Ligas a redes sociales (Facebook, Twitter, etc.) & 11 & 84.6 & 4 & 80 & 3 & 75 & 18 & 81.8 \\
\hline
\end{tabular}

Fuente: Elaboración propia basada en análisis de los sitios webs de los partidos políticos con representación parlamentaria. Información actualizada a junio de 2012.

14 http://www.partidogen.com.ar/campana.php

15 Frente para la Victoria y Partido Justicialista (que para efectos de nuestras clasificación aparecen juntos, aunque tienen dos sitios webs), Pro, Partido Socialista, Nuevo Encuentro y Generación para un Encuentro Nacional. 


\section{El uso de los medios digitales por parte de los parlamentario/as ${ }^{16}$}

Con respecto a la presencia en línea a través de webs, blogs, Facebook y Twitter, se observó que un tercio de los diputados argentinos y uruguayos han abierto su sitio web o blog (30 y 32\%, respectivamente), pero que sólo el $6 \%$ de los representantes paraguayos lo han hecho. Los diputados argentinos son los que tienen mayor cantidad de cuentas de Twitter ${ }^{17}$ (53\%), y es el medio más popular entre ellos. Los diputados paraguayos y uruguayos se inclinan por los perfiles de Facebook (49 y 69\%, respectivamente). Sin embargo, Twitter no deja de ser popular entre los segundos, casi la mitad de los diputados posee cuenta (46\%); en Argentina sucede algo similar con las preferencias por los perfiles de Facebook (44\%). La presencia de los diputados paraguayos en Twitter es excepcional, apenas el 10\% tiene cuenta (nueve diputados). Finalmente, la utilización de páginas de Facebook está muy extendida entre los representantes argentinos: registran setenta páginas activas (27\%), lo que no ocurre en Uruguay y Paraguay (4 y 3\%, respectivamente). Una observación comparada entre perfiles, páginas $\mathrm{y}$ cuentas, muestra que veinte diputados argentinos tienen los tres espacios (y ocho tienen además página web o blog). De los titulares de las setenta páginas, sólo diez no tienen cuenta en Twitter. En Paraguay sólo un diputado tiene perfil, página, blog y cuenta en Twitter, mientras que en Uruguay en ningún caso se cumplen las tres condiciones (tabla 3).

En definitiva, mientras en el análisis del despliegue de webs por partidos políticos no encontramos relación entre la difusión de Internet en el territorio y la adopción de tecnología, en el análisis de la adopción individual sí parece haber vínculos entre una mayor difusión y actividad en línea y una mayor presencia de los políticos en las redes sociales (hipótesis a).

Tabla 3: Diputado/as que tienen sitios web o cuentas en redes sociales

\begin{tabular}{lc|c|c|c|c|c|c|c}
\hline & \multicolumn{2}{c}{ Argentina } & \multicolumn{2}{c}{ Paraguay } & \multicolumn{2}{c}{ Uruguay } & \multicolumn{2}{c}{ Total } \\
\hline Sitio web personal o blog & 77 & $30 \%$ & 5 & $6 \%$ & 32 & $32 \%$ & 114 & $26 \%$ \\
Perfil de Facebook (Fb) & 112 & $44 \%$ & 39 & $49 \%$ & 68 & $69 \%$ & 219 & $50 \%$ \\
Página de Facebook (Fbp) & 70 & $27 \%$ & 3 & $4 \%$ & 2 & $2 \%$ & 75 & $17 \%$ \\
Cuenta de Twitter (Tw) & 137 & $53 \%$ & 9 & $11 \%$ & 45 & $46 \%$ & 191 & $44 \%$ \\
\hline Total de casos observados & 257 & $100 \%$ & 80 & $100 \%$ & 99 & $100 \%$ & 436 & 100 \\
\hline
\end{tabular}

Fuente: Elaboración propia. Recolección de datos: febrero-abril de 2012.

16 Nuestra investigación incluye una revisión de los sitios web de gobierno, partidos políticos, medios masivos de comunicación más populares, bases de datos elaboradas por ONG y otras organizaciones de la sociedad civil, así como búsquedas en Wikipedia y Google.

17 En Argentina se identificaron 150 cuentas de Twitter de las cuales 137 se han considerado activas, con al menos un tweet desde su creación. El análisis en este caso se realiza sobre la base de los casos activos. 


\section{¿Quiénes están en Facebook y Twitter?}

Los datos muestran que — salvo algunos matices - no hay marcada diferenciación entre diputados y diputadas con presencia en redes sociales. La brecha de género es un dato que no se debe desconocer, evidente en la cantidad de bancas que ocupan hombres y mujeres (en Uruguay y Paraguay el 88\% están ocupadas por hombres, Argentina presenta un panorama más equitativo, con un $37 \%$ de diputadas). En términos relativos, las parlamentarias tienen en $\mathrm{Pa}-$ raguay mayor presencia en los perfiles de Facebook que sus pares masculinos ( $80 \%$ vs. $44 \%$ ), lo cual, con menor distancia, sucede también en Argentina. En Uruguay, a su vez, pertenecen a diputadas las únicas dos páginas de Facebook registradas (el caso contrario sucede en Paraguay, donde las páginas son de diputados) (tabla 4).

Tabla 4. Género y edad de los diputado/as que tienen perfil/página de Facebook y cuenta en Twitter

\begin{tabular}{|c|c|c|c|c|c|c|}
\hline & & \multicolumn{2}{|c|}{ Hombres } & \multicolumn{2}{|c|}{ Mujeres } & \multirow{2}{*}{$\begin{array}{c}\text { Edad } \\
\text { (promedio) }\end{array}$} \\
\hline & & $N$ & $\%$ & $N$ & $\%$ & \\
\hline \multirow{4}{*}{ AR } & Diputado/as (total) & 161 & 63 & 96 & 37 & $54(1)$ \\
\hline & Perfil de Facebook & 68 & 42 & 44 & 46 & 53 \\
\hline & Página de Facebook & 50 & 31 & 20 & 21 & 52 \\
\hline & Cuenta de Twitter & 91 & 56 & 46 & 48 & 53 \\
\hline \multirow{4}{*}{ PY } & Diputado/as (total) & 70 & 87 & 10 & 12 & $49(2)$ \\
\hline & Perfil de Facebook & 31 & 44 & 8 & 80 & 48 \\
\hline & Página de Facebook & 3 & 4 & 0 & 0 & 48 \\
\hline & Cuenta de Twitter & 8 & 11 & 1 & 10 & 44 \\
\hline \multirow{4}{*}{ UY } & Diputado/as (total) & 87 & 88 & 12 & 12 & $52(3)$ \\
\hline & Perfil de Facebook & 60 & 69 & 8 & 67 & 51 \\
\hline & Página de Facebook & 0 & 0 & 2 & 16 & 60 \\
\hline & Cuenta de Twitter & 39 & 45 & 6 & 50 & 49 \\
\hline
\end{tabular}

Nota: Información sobre género, disponible para 100\% de cada caso. Información de edad disponible: Perfil de Facebook Ar., 75\%; Py., 72\%; Uy., 94\%. Página de Facebook: Ar., 81.4\%; Py., 100\%; Uy., 100\%. Cuenta de Twitter: Ar., 78.7\%; Py., 100\%; Uy., 97.8\%. (1) Válido 70.4\%. (2) Válido 62.9\%, (3) Válido Uy., 88.9\%.

Fuente: Elaboración propia. Datos actualizados a mayo de 2012.

En cuanto a la edad, en casi todos los casos el promedio es menor al de la edad de la cámara. La excepción son los que han abierto páginas de Facebook en Uruguay: mientras el promedio de la cámara es de 52 años, los diputados con Facebook presentan una media de sesenta años. En Paraguay, los diputados con cuenta de Twitter ( 44 años) están por debajo de la media de la cámara (49 años), aunque en este caso más de la mitad tiene menos de cuarenta años. 
Finalmente, concuerda con los promedios generales el nivel educativo alcanzado (universitario) por los diputados usuarios de redes.

\section{Más Superman que Clark Kent: el uso público (político) de las redes sociales}

En el caso de Twitter sólo una de las 191 cuentas analizadas está cerrada, mientras que los perfiles de Facebook se encuentran en su mayoría abiertos o parcialmente abiertos; el caso paraguayo es el que presenta el mayor porcentaje de cuentas abiertas (73\%). ${ }^{18}$ Las cuentas cerradas que formaron parte de este estudio no reunían las características para identificarse como de uso político (foto de perfil, referencia a actividad política, etc.). Además, el cerrar una cuenta o un perfil sugiere intencionalidad, lo que puede vincularse con la preservación de un ámbito privado. Los perfiles de Facebook son abiertos por defecto, lo que lleva a suponer que su cierre muestra tanto el conocimiento como la intencionalidad de mantener la presencia virtual en lo privado. Sin embargo, no todas las cuentas abiertas muestran un uso claramente político (en algunos casos se ven pocas referencias a la actividad política, y más bien priman los comentarios entre los amigos del usuario — que en general son pocos—, las fotografías familiares o las referencias a actividades privadas).

En cambio, el análisis de las páginas de Facebook sugiere que allí existe un mayor conocimiento de la potencialidad de las redes sociales para el quehacer político. Si bien unas páginas son más completas que otras, el formato de presentación claramente se orienta a destacar la actividad política del usuario con referencias al partido o figuras políticas fácilmente identificables. En Argentina, por ejemplo, la presidenta Cristina Fernández, el expresidente Néstor Kirchner, Eva Duarte (Evita) y Juan Domingo Perón son las figuras a las que más se apela. Nuestro estudio sugiere que en las páginas se muestra una mayor distancia entre el representante (administrador de la página) y quienes le siguen, dado que la mayor parte de la interacción sucede entre los seguidores o miembros del página, mientras que el administrador se limita a postear información, noticias, su agenda o anunciar sus actividades en tiempo real, mostrando así un clásico patrón de interacción unidireccional. En ocasiones, incluso sugieren que la cuenta o perfil no es mantenida por el político titular de la misma.

Retomando de los comics las figuras de Clark Kent y Superman como metáfora de la disociación-complementaria entre las vidas privada (Kent) y pública

18 Accesibilidad en las cuentas de Facebook (abierta / parcialmente abierta / cerrada); AR: 65.5\%, 19\%, 15.5\%; PY: 73.3\%, 22.2\%, 4.4\%; UY: 68.4\%, 26.3\%, 5.3\%.

Y. Welp, A. Marzuca | Presencia de partidos políticos y diputados en Internet en Argentina, Paraguay y Uruguay Perfiles Latinoamericanos, 24(47) | FLACso México | pp. 199-224 | DoI: 10.18504/pl2447-011-2016 
(Superman) de los políticos para caracterizar su utilización de las redes sociales, diríamos que en su gran mayoría, los diputados tienden a usar políticamente (público) su presencia en la redes. Puesto que, aunque la gama de situaciones abarca desde el uso de aplicaciones y posibilidades básicas (muy cercano a un manejo personal) hasta productos complejos que combinan varias herramientas y que dan cuenta de una estrategia comunicacional programada, los contenidos, uso y el sentido de su mensaje y presencia están altamente vinculados a su faceta pública.

Algunas explicaciones sobre las diferencias en el uso de los medios digitales entre los políticos se relacionan con el know-how y la planificación estratégica. Por know-how referimos los casos en los que las personas muestran la intención de desarrollar las capacidades sobre cómo utilizar los medios digitales e incluir este conocimiento en su actividad política. Estos son los que presentan un uso más sofisticado de los medios digitales (combinando Facebook, Twitter y blogs, integrando nuevos dispositivos tecnológicos, encargándose personalmente, o encargando a colaboradores especializados, del mantenimiento de un perfil, página o cuenta actualizada). También se hallaron casos en los que los políticos utilizan los medios digitales como parte de un plan de acción que responde a una estrategia general de su grupo político. Esto se constata con la existencia de líderes partidarios que promueven su uso entre los miembros del partido (organizando talleres de entrenamiento, o contratando asesores de marketing político expertos en el manejo de estos dispositivos). ${ }^{19}$

\section{Difusión y popularidad}

La popularidad de diputados y diputadas en Facebook se midió considerando la cantidad de amigos de los perfiles y el número de suscriptores de las páginas. En Argentina y Uruguay, la mayoría de los perfiles (76\%, en ambos casos) tienen una popularidad media-alta (entre 501 y 5000); en Paraguay la tendencia es entre baja y media (entre 1 y 2000 amigos). ${ }^{20}$ Se encontró además a diputados que tienen más de un perfil en Facebook: cinco en Argentina y Paraguay ( 4.5 y $12.9 \%$ sobre el total, respectivamente) y siete en Uruguay (10.3\%). Esto parece relacionarse al grado de conocimiento o apropiación de las posibilidades para operar en las redes sociales. Muchos parlamentarios con perfiles ya creados, al llegar al límite máximo de cinco mil amigos abren un nuevo perfil dispersando

19 Véase, por ejemplo, la estrategia del Partido Colorado en http://www.partidocolorado.com.uy/noticias/ Colorados-2.0/71

20 Categorías de popularidad de perfiles: 1- 500 (baja), 501-2000 (media), 2001-5000 (alta). 
su información. También se registraron casos donde la invitación o redireccionamiento es hacia una página.

La popularidad de las setenta páginas argentinas varía entre los 4 y los 43510 "me gusta" y su promedio se ubica en los 4483. Más de la mitad de las dieciséis páginas más populares (que tienen más de cinco mil seguidores) tienen al menos doce mil "me gusta". En Uruguay, las dos páginas registradas tienen 5621 y 3858 suscriptores. Finalmente, la página paraguaya más popular tiene 1431, mientras que la menos popular alcanza los 43 .

Como ya se ha dicho, Twitter es el medio más utilizado por los diputados argentinos. Pertenecientes a 150 diputados, se registraron 152 cuentas, entre las que se halla la más antigua (fechada en enero de 2009). La gran mayoría (137 de 139) tiene al menos un tweet desde su apertura. Por su parte, la cuenta más antigua en Paraguay está fechada en octubre de 2009, y en Uruguay, en enero de 2010. A su vez, en Paraguay y Uruguay la mayoría de las cuentas se crearon en 2011 mientras que en Argentina remiten a 2010. Esto sugiere que la presencia individual en Twitter se inició antes en Argentina, pese a que las webs partidarias muestran estar menos desarrolladas en este país.

La popularidad de las cuentas de Twitter de los diputados, medida por el número de seguidores, muestra marcadas diferencias entre y al interior de los casos. En Argentina, la cuenta más popular contaba al momento del análisis (abril de 2012) con más de 157 mil seguidores (Francisco de Narváez, @denarvaez, Frente Peronista). En Paraguay, algo más de diez mil (Sebastián Acha $@$ @ebAcha, Partido Patria Querida) y en Uruguay, poco más de cinco mil (Luis Lacalle Pou, @luislacalle400). Las cuentas menos populares se registran en Argentina y Uruguay, con cuatro seguidores, y pertenecen ambas al partido con mayor cantidad de bancas en su respectivo país. ${ }^{21} \mathrm{El}$ número promedio de seguidores de cuentas de Twitter de parlamentarios en Argentina es de 7613, en Paraguay de 1536 y en Uruguay de 742.

Por último, se observó también la intensidad de uso de Twitter, midiéndolo como el número de publicaciones que un parlamentario realiza en un periodo determinado (desde la creación de la cuenta hasta abril de 2012). La mayoría presentó un uso bajo o intermedio (tabla 7). En Argentina, el promedio de publicaciones es de 16 tweets por día, en Paraguay de once, y en Uruguay de nueve. Sin embargo, los promedios pueden ocultar un uso errático debido a que muchos diputados muestran una intensa actividad en unos días determinados y luego no hay actividad por varios días (por ejemplo, 131 tweets en un día, @Carlos_Kunkel, el 14 de marzo de 2012).

21 Argentina: Fernando Yarade (@FernandoYarade); Frente para la Victoria. Uruguay: Oscar Groba (@GrobaOscar), Frente Amplio.

Y. Welp, A. Marzuca | Presencia de partidos políticos y diputados en Internet en Argentina, Paraguay y Uruguay Perfiles Latinoamericanos, 24(47) | FLACso México | pp. 199-224 | DoI: 10.18504/pl2447-011-2016 
Tabla 5. Intensidad en el uso de Twitter

\begin{tabular}{lcccccc}
\hline & \multicolumn{2}{c}{ Argentina } & \multicolumn{2}{c}{ Paraguay } & \multicolumn{2}{c}{ Uruguay } \\
\hline Nivel de uso & $N$ & $\%$ & $N$ & $\%$ & $N$ & $\%$ \\
\hline Intensivo ( 8 y más) & 7 & 5 & 1 & 11 & 0 & 0 \\
Alto ( 4-7) & 5 & 4 & 1 & 11 & 2 & 4 \\
Intermedio ( 1-3) & 38 & 28 & 3 & 33 & 14 & 31 \\
Bajo ( menos de 1) & 87 & 64 & 4 & 45 & 29 & 65 \\
\hline
\end{tabular}

Fuente: Elaboración propia.

Si bien es necesario profundizar en el análisis del contenido de la comunicación entre políticos y seguidores, amigos o fans, la lectura general anuncia pautas relevantes en lo que refiere al perfil de la participación de los políticos en estas redes. Destacan dos aspectos que dan cuenta de una marcada asimetría entre políticos y seguidores. Por una lado, se replica en estos espacios la lógica emisorreceptor, basada en una circulación de mensajes principalmente unidireccional (desde los políticos hacia sus seguidores). La interacción — cuando aparece- se realiza en general entre pares políticos o con otros actores reconocidos del ámbito público (político-político, político-periodista, político-actor social). A su vez, y especialmente en lo que se refiere al uso de Twitter, los políticos suelen "seguir menos de los que los siguen", y suelen seguir a otros políticos (nacionales y extranjeros), figuras públicas (sindicales, empresariales, analistas, artistas, deportistas e instituciones deportivas) y portales de noticias. Los miembros de la red no-políticos (seguidores), por su parte, tienden a amplificar el mensaje comentando la postura personal en sus propios espacios, más que a buscar el diálogo con el político. La combinatoria de estas observaciones estaría echando por tierra la expectativa de contar con un diálogo de proximidad, favorecido por el potencial de horizontalidad de estos espacios.

\section{La actividad de los parlamentarios por partidos}

Aunque los partidos con mayor cantidad de escaños en general son los que también tienen el mayor número de diputados que utilizan medios digitales (tabla 6), no hay una relación lineal entre una dimensión y otra. Por ejemplo, el Frente Para la Victoria (FPv) concentra el 53\% de los escaños y el 42\% de las cuentas de Twitter en Argentina; en Paraguay, el Partido Liberal Radical Auténtico cuenta con el 36\% de los escańos, pero ni uno solo de sus diputados tiene página de Facebook; mientras que, en Uruguay, el Partido Nacional, con el 30\% de escaños, reúne el $50 \%$ de las páginas de Facebook y el 48\% de las cuentas de Twitter sobre el total de diputados de la Cámara. El panorama es más hetero- 
géneo al analizar la popularidad de cuentas de Facebook por partido (véase más arriba). En Uruguay hay cierto predominio de un partido en cada extremo, ${ }^{22}$ mientras que tanto en Argentina como en Paraguay, primeras y últimas posiciones muestran una gama variada de actores. No obstante, el partido con mayor cantidad de escaños en ningún caso es el que registra las cuentas más populares, por el contrario, diputados de partidos mayoritarios son los que tienen en los tres casos las cuentas con menor cantidad de seguidores. ${ }^{23}$

Tabla 6. Porcentaje de diputados por partido político y porcentaje sobre el total de perfiles/páginas de Facebook y cuentas de Twitter

\begin{tabular}{lrrrrr}
\hline \multicolumn{1}{c}{ Partido político } & Total & \multicolumn{1}{c}{$\%$} & Perf. Fb \% & Pág. Fb\% & Twitter \% \\
\hline Frente para la Victoria y aliados (FPV) & 136 & 52.95 & 47 & 44 & 42 \\
Unión Cívica Radical y aliados (UCR) & 41 & 15.95 & 19 & 16 & 19 \\
Frente Amplio Progresista (FAP) & 22 & 8.56 & 11 & 11 & 9 \\
AR* $\quad$ Frente Peronista (FP) & 22 & 8.56 & 8 & 6 & 9 \\
PRO & 13 & 5.06 & 5 & 10 & 9 \\
Coalición Cívica - ARI (CC.CC- ARI) & 6 & 2.33 & 5 & 3 & 2 \\
Otros & 17 & 6.61 & 5 & 10 & 8 \\
Asociación Nacional Republicana-Partido Colorado (ANR) & 33 & 41.25 & 33 & 67 & 56 \\
Partido Liberal Radical Auténtico (PLRA) & 29 & 36.25 & 36 & 0 & 11 \\
Partido Patria Querida (PPQ) & 4 & 5.00 & 5 & 33 & 11 \\
Partido Unión Nacional de Ciudadanos Éticos (PUNACE) & 12 & 15.00 & 20 & 0 & 22 \\
Partido Democrático Progresista (PDP) & 1 & 1.25 & 3 & 0 & 0 \\
Movimiento de Participación Ciudadana (MPC) & 1 & 1.25 & 3 & 0 & 0 \\
Frente Amplio (FA) & 50 & 50.50 & 44 & 50 & 24 \\
Partido Nacional (PN) & 30 & 30.30 & 34 & 50 & 48 \\
Partido Colorado (PC) & 17 & 17.17 & 20 & 0 & 29 \\
Partido Independiente (PI) & 2 & 2.02 & 1 & 0 & 0 \\
\hline
\end{tabular}

* Para el caso de Argentina, se optó por realizar una agrupación propia de los partidos políticos, basada en la forma en que aparecen organizados en el sitio web del Parlamento y en la información que ofrecen los parlamentarios en sus sitios webs y/o cuentas en las redes sociales. Así, Frente para la Victoria y aliados (Frente para la Victoria-PJ, 116; Nuevo Encuentro, 5; Movimiento Popular Neuquino, 4; Frente Cívico por Santiago, 7; Partido Justicialista de la Pampa, 2; Renovador de Salta, 1; Frente Peronista Federal, 1); Unión Cívica Radical y aliados (Unión Cívica Radical, 40; UDESO, 1); Frente Amplio Progresista (Unidad Popular, 5; Libres del Sur, 1; Partido Socialista, 6; Generación para un Encuentro Nacional, GEN, 5; Frente Cívico-Córdoba, 5); Frente Peronista (Frente Peronista, 21; Socialistas del MIJD, 1); PRO (Demócrata de Mendoza, 2; Propuesta Republicana, 11); Coalición Cívica-ARI, 6; Otros (Unión Peronista, 3; Córdoba Federal, 2; Corriente de Pensamiento Federal, 1; Democracia Igualitaria y Participativa, 1; Unidad para el Desarrollo Social y la Equidad, 1; Demócrata Progresista, 1; Movimiento Proyecto Sur, 3; Partido Federal Fueguino, 1; Salta Somos Todos, 1; Santa Fe en Movimiento, 1; Unión por Todos, 1; Unión por San Juan, 1).

Fuente: Elaboración propia. Datos actualizados a mayo de 2012.

22 Las tres cuentas más populares (en orden descendente: @luislacalle400, @AnaLiaPineyrua y @dipjgarcia) pertenecen al PN. Dos de las tres con menor cantidad de seguidores (orden creciente: @GrobaOscar y @ipassada) son de miembros del FA.

23 Argentina: Fr, Pro y Movimiento Proyecto Sur (más populares en orden decreciente: @denarvaez, @gabimichetti, @fernandosolanas). Fpv-PJ y UCR (menos populares en orden creciente: @FernandoYarade, @LuisCigogna, @DipLindaYague). Paraguay: PPQ, ANR, PunAce (más populares en orden decreciente: @SebAcha, @oscartuma, @FabiolaOviedo); ANR y punAce (menos populares en orden creciente: (@justocardenas, @VictorYambay, @oviedo_ariel). 
En Argentina, por ejemplo, todos los diputados del pro (13 de 257) tienen cuentas de Twitter, mientras que un alto porcentaje también es usuario de Facebook (54\% tiene páginas, 46\% tiene perfil). En el caso uruguayo, para el Partido Colorado (17 de 99 asientos) se identificó que el 82\% de sus diputados tiene perfil de Facebook y el 77\% cuenta de Twitter. Mientras que, en Paraguay, los dos diputados de los partidos más pequeños tienen perfiles de Facebook, y es el Partido Patria Querida el que tiene los porcentajes más altos de presencia en redes sociales (tabla 7).

En cuanto a la ideología, se observó que no hay una relación clara (por ejemplo, el centroizquierda Frente para la Victoria y el pro de centroderecha son los más activos en las redes en Argentina). El análisis sugiere que más que la ideología el liderazgo podría explicar por qué el Frente Amplio (centroizquierda) tiene menor presencia relativa en las redes que el Partido Colorado (centroderecha).

Tabla 7. Distribución de perfiles/páginas de Facebook y cuentas de Twitter al interior de los partidos

\begin{tabular}{|c|c|c|c|c|c|}
\hline & Partido político & Dip. & $\% F b$ & $\% F b p$ & $\% T w$ \\
\hline \multirow[t]{7}{*}{ AR } & Frente para la Victoria y aliados (FPV) & 136 & 39 & 23 & 43 \\
\hline & Unión Cívica Radical y aliados (UCR) & 41 & 51 & 27 & 63 \\
\hline & Frente Amplio Progresista (FAP) & 22 & 55 & 36 & 59 \\
\hline & Frente Peronista (FP) & 22 & 41 & 18 & 59 \\
\hline & PRO & 13 & 46 & 54 & 100 \\
\hline & Coalición Cívica - ARI (CC.CC- ARI) & 6 & 83 & 33 & 50 \\
\hline & Otros & 17 & 35 & 41 & 65 \\
\hline \multirow[t]{6}{*}{ PY } & Asociación Nacional Republicana- Partido Colorado (ANR) & 33 & 39 & 6 & 15 \\
\hline & Partido Liberal Radical Auténtico (PLRA) & 29 & 48 & 0 & 3 \\
\hline & Partido Patria Querida (PPQ) & 4 & 50 & 25 & 25 \\
\hline & Partido Unión Nacional de Ciudadanos Éticos (PUNACE) & 12 & 67 & 0 & 17 \\
\hline & Partido Democrático Progresista (PDP) & 1 & 100 & 0 & 0 \\
\hline & Movimiento de Participación Ciudadana (MPC) & 1 & 100 & 0 & 0 \\
\hline \multirow[t]{4}{*}{ UY } & Frente Amplio (FA) & 50 & 60 & 2 & 22 \\
\hline & Partido Nacional (PN) & 30 & 77 & 3 & 70 \\
\hline & Partido Colorado (PC) & 17 & 82 & 0 & 77 \\
\hline & Partido Independiente (PI) & 2 & 50 & 0 & 0 \\
\hline
\end{tabular}

\section{Conclusiones}

Una vez completado el recorrido, podemos extraer algunas conclusiones. En primer lugar, se observa una difusión considerable de webs y redes sociales para la actividad política. Sin embargo, esta adopción muestra importantes diferencias tanto entre países como entre partidos y, al interior de los partidos, entre representantes. 
Sólo el 53.6\% de los partidos con representación en la Cámara de Diputados de Argentina, Paraguay y Uruguay tienen sitios web. La observación entre los casos sugiere que un partido institucionalizado tiene mayores incentivos y recursos para invertir en su presencia en línea. Así, mientras en Uruguay todos los partidos cuentan con página web, en Paraguay la mayoría cuenta con ella (83\%) y en Argentina menos de la mitad la poseen (42\%). Por el contrario, mayor institucionalización y una mejor posición en el espectro electoral parecerían inhibir usos innovadores de la Web. Con contadas excepciones, las webs analizadas apenas incluían alguna información (organización, objetivos, autoridades), ignorando o rechazando las posibilidades abiertas por estos nuevos medios de comunicación (captación de recursos, interacción con los electores, atracción de nuevos adherentes o seguidores), mientras que el uso de corte innovador se restringe a partidos pequeños. Los hallazgos, por lo tanto, son similares a los de estudios centrados en casos europeos y estadounidenses, en los que en general los partidos utilizan sus sitios webs para publicar información básica, a la vez que mantienen estrategias comunicacionales tradicionales y procesos jerárquicos en la toma de decisiones. También en consonancia con estos estudios, se ha encontrado los usos más novedosos en los partidos pequeños.

El panorama es más diverso en el terreno individual, donde se revela que ni el género ni la orientación ideológica son determinantes para el posicionamiento en los nuevos medios digitales. Sin embargo, entre los países hay diferencias relevantes. Los diputados y diputadas argentinas son los principales usuarios de Twitter y los que más recurren a las páginas de Facebook, insinuando mayor conocimiento o explotación de las posibilidades de las redes sociales para la actividad política. En Uruguay y aún más en Paraguay hay mayor cantidad de perfiles de Facebook asociados a la vida privada. De todas maneras, la diferencia en la utilización de Twitter entre Argentina (53\%) y Uruguay (46\%) permite ubicarlos con ciertos patrones similares en materia de difusión que los despegan de la imagen que hoy arroja el caso paraguayo (9\%). Si se tuviera que construir una escala que ordena los tres casos de acuerdo a difusión, popularidad y uso de redes sociales, el orden sería Argentina, Uruguay y Paraguay.

Las posibilidades abiertas por los distintos medios permiten conectar los espacios virtuales y de esta forma potenciar la comunicación y difusión de ideas: por ejemplo, al publicar una propuesta de ley en un blog o página web se puede anunciar en Twitter y Facebook remitiendo a la liga del blog o página web para la lectura completa. Estos usos más complejos de los medios digitales aún son escasamente utilizados por la mayoría de los políticos. De la gama de herramientas analizadas, parece haber una clara diferencia entre el uso de Facebook y el de Twitter, dado que se encontraron más casos de uso personal o no profesional en Facebook, mientras que aquellos con cuentas en Twitter tienden 
a darles un mayor uso político. Asimismo, en Facebook se halló una importante diferencia entre el uso de los perfiles y las páginas: si bien algunos perfiles de los representantes tienen un contenido altamente orientado a su actividad personal, las páginas presentan un claro uso político.

La creciente difusión de Internet y las redes sociales permite pensar que no hay vuelta atrás en su inclusión como nuevos medios de comunicación entre ciudadanos y entre políticos y ciudadanos. En este sentido, se observa una tendencia indiscutible. A la vez, aunque no aparecen cambios sustanciales que indiquen que la lógica horizontal y multinodular de los medios digitales y en particular de las redes sociales esté siendo adoptada y explotada por los partidos, sí parece ser una herramienta de base individual. Los políticos se ven provistos de un canal de expresión directa en tiempo real que, por un lado, puede replicarse a través de la llegada a los otros medios de corte tradicional y, por otro, sitúa una nueva arena para la discusión entre sus pares. Estos dos aspectos, que surgen de una lectura general del uso y los contenidos, dejan la impresión de que la interacción directa con los ciudadanos — cuando sucede- ocupa un muy distante tercer lugar.

\section{Referencias}

\section{Bibliohemerografía}

Anduiza, E., Jensen, M. J. \& Jorba, L. (2012). Digital Media and Political Engagement Worldwide. A Comparative Study. Cambridge: Cambridge University Press.

Araya Moreno, E. \& Barría Traverso, D. (2009). e-Participación en el senado chileno: ‘aplicaciones deliberativas? Convergencia, 16(51), 239-268.

Breuer, A. \& Welp, Y. (2014). Re-assessing ICTs for Democratic Governance in Latin America. En Breuer, A. \& Welp, Y., Digital Technologies for Democratic Governance in Latin America (pp. 217-223). Londres-Nueva York: Routledge.

Borge, R. (2005). La participación electrónica: estado de la cuestión y aproximación a su clasificación. Internet, Derecho y Politica (1). Recuperado de http://www.uoc.edu

Burch, S. \& Osvaldo León (2014). Social movements, democratic participation and ICTs. En Breuer, A. \& Welp, Y., Digital Technologies for Democratic Governance in Latin America (pp. 127-145). Londres-Nueva York: Routledge.

Calderaro, A. (2014). Internet Politics Beyond the Digital Divide. En Patrut, B. \& Patrut, M. (Eds.), Social Media in Politics: Case Studies on the Political Power of Social Media (pp. 3-17). Nueva York: Springer International Publishing. 
Castells, M. \& Sey, A. (2004). The Internet and the Political Process. En Castells, M. (Ed.), The Network Society: A Cross-Cultural Perspective. Northamption: Edward Elgar Publication.

Dader, J. L. (2009). Ciberpolítica en los websites de partidos políticos. La experiencia de las elecciones de 2008 en España ante las tendencias transnacionales. Revista de Sociología e Politica, 17(34), 45-62.

Dahl, R. (1989). Democracy and Its Critics. Yale: Yale University Press.

Dalton, R. \& Weldom, S. (2007). Partisanship and Party System Institutionalization. Party Politics, 13(2), 179-196.

De Rosa, R. (2013). The Five Stars Movement in the Italian Political Scenario. A Case for Cybercratic Centralism? Journal of eDemocracy, 5(2).

Frick, M. (2005). Parliaments in the Digital Age. Exploring Latin America. e-Working Papers 2005/01. e-Democracy Centre. Recuperado de www.edemocracycentre.ch

Fumega, S. \& Scrollini, M. (2012). El acceso a la información y los gobiernos abiertos en América Latina. En La promesa del gobierno abierto. Recuperado de http://www.lapromesadelgobiernoabierto.info/index.php

Gibson, R. \& Ward, S. (2000). A Proposed Methodology for Studying the Function and Effectiveness of Party and Candidate Web Sites. Social Science Computer Review, 3(18), 301-319.

Grant, W., Moon, B. \& Busby Grant, J. (2010). Digital Dialogue? Australian Politicians' Use of the Social Network toll Twitter. Australian Journal of Political Science, 45(4).

Hague, B. \& Loader, B. D. (1999). Digital Democracy. Discourse and Decision Making in the Information Age. Londres: Routledge.

Hargittai, E. (1999). "Weaving the Western Web: Explaining Differences in Internet Connectivity among OECD Countries. Telecommunications Policy, 23, 701-718.

Hartleb, F. (2013). Anti-Elitist Cyber Parties? Journal of Public Affairs, 13(4), 355-369.

Howard, P. (2005). Deep Democracy, Thin Citizenship: The Impact of Digital Media in Political Campaign Strategy. The ANNALS of the American Academy of Political and Social Science, 597, 153-170.

Hunter, M. (2011). Mapping Digital Media, Reference. Series (2). 
Kamarck, E. (1999). Campaigning on the Internet in the Elections of 1998. En Kamarck, E. \& Nye, J. (Eds.), Democracy.com? Governance in a Networked World. Hollis, NH: Hollis.

López, S. (2005). Partidos desafiantes en América Latina: representación política y estrategias de competencia de las nuevas oposiciones. Revista de Ciencia Politica, 25(2), 37-64.

Loza, N. (Ed.) (2011). Voto electrónico y democracia directa. Los nuevos rostros de la política en América Latina. México: Flacso México.

Lusoli, W., Ward, S. \& Gibson, R. (2006). (Re)connecting politics? Parliament, the Public and the Internet. Parliamentary Affairs, 59(1), 24-42.

Llorente, J. A. \& Cuenca, O. (2014). Elecciones Brasil 2014: la reputación de los pre-candidatos presidenciales en Twitter: un análisis multidimensional. Informe Especial I+D Consultores. Recuperado de http://www.dmasillorenteycuenca.com/publico/140520_dmasi_Informe_ Especial_elecciones\%20en\%20brasil_ESP.pdf

Mainwaring, S. \& Scully, T. (1995). Introduction: Party Systems in Latin America. En Mainwaring, S. \& Scully, T. (Eds.), Building Democratic Institutions: Party Systems in Latin America. Stanford: Stanford University Press.

Marzuca Perera, A., Serdült, U. \& Welp, Y. (2011, primavera). Questâo Pública: First Voting Advice Application in Latin America. En Tambouris, E., Macintosh, A. \& De Bruijn, H. (Eds.) Electronic Participation: Third IFIP WG 8.5 International Conference, ePart 2011, Delft. The Netherlands, August 29-September 1, Proceedings [Lecture Notes in Computer Science 6847]. Heidelberg, 216-227.

Musiani, F. (2013). Avant-Garde Digital Movement or "Digital Sublime" Rhetoric. En Patrut, B. \& Patrut, M. (Eds.), Social Media in Politics: Case Studies on the Political Power of Social Media. Nueva York: Springer International Publishing.

Nixon, P. \& Johansson, H. (1999). Transparency Through Technology: The Internet and Political Parties. En Hague, B. \& Loader, B. (Eds.), Digital Democracy: Discourse and Democracy in the Information Age. Londres: Routledge.

Norris, P. (2001). Digital Divide. Civic Engagement, Information Poverty, and the Internet Worldwide. Cambridge: Cambridge University Press.

Padró-Solanet, A. \& Cardenal, A. S. (2008). Partidos y política en Internet: Un análisis de los websites de los partidos políticos catalanes. IDP. Revista de Internet, Derecho y Política, (6).

Römmele, R. (2003). Political Parties, Party Communication and New Information and Communication Technologies, Party Politics, 9, 7-20. 
Setälä, M. \& Gronlünd, K. (2006). Parliamentary Websites: Theoretical and Comparative Perspectives. Information Polity, (11), 149-162.

Socialbakers Statistics (2012) Facebook Statistics 2012: Top Growing Countries. Disponible en http://www.socialbakers.com/blog/684-facebook-statistics-2012-top-growing-countries

Sæbø, Ø. (2011). Understanding Twitter TM Use among Parliament Representatives: A Genre Analysis. En Tambouris M. \& De Bruijn (Eds.), Electronic Participation Third IFIP WG 8.5 International Conference, ePart 2011, Delft, The Netherlands, August 29-September 1.

Túñez, M. \& Sixto, J. (2011). Redes sociales, política y Compromiso 2.0: La comunicación de los diputados españoles en Facebook. Revista Latina de Comunicación Social, (66), 1-25.

Trechsel, A., Kies, R., Mendez, F. \& Schmitter, P. (2003). Evaluation of the Use of new technologies in order to facilitate democracy in Europe. eDC Working Papers.

Welp, Y. \& Wheatley, J. (2009). The effect of ICTs and new media on Political Party Systems: more democracy or more populism? Paper prepared for the ECPR General Conference, Postdam.

Welp, Y. (2011). Bridging the Political Gap? The adoption of ICTs for the improvement of Latin American Parliamentary democracy. En Sobaci, Z. (Ed.), E-Parliament and ICT-Based Legislation: Concept, Experience and Lessons. IGI Global Publisher.

Welp, Y. (2008). América Latina en la era del gobierno electrónico. Análisis de la introducción de nuevas tecnologías para la mejora de la democracia y el gobierno. Revista del CLAD Reforma y Democracia, (41).

Welp, Y. \& Marzuca, A. (2014). South American Politics in the Information Age. A Study of Political Parties and MPs on the net in Argentina, Paraguay and Uruguay. En Breuer \& Welp (Eds.), Digital Technologies for Democratic Governance in Latin America: Opportunities and Risks. uK: Routledge.

\section{Sitios web de los partidos políticos ${ }^{24}$}

\section{Argentina}

www.frenteparalavictoria.org: Frente para la Victoria - PJ

www.ucr.org.ar: Unión Cívica Radical

24 Los sitios web citados en esta lista se visitaron por última vez en octubre de 2012.

Y. Welp, A. Marzuca | Presencia de partidos políticos y diputados en Internet en Argentina, Paraguay y Uruguay Perfiles Latinoamericanos, 24(47) | FLACso México | pp. 199-224 | DoI: 10.18504/pl2447-011-2016 
www.frenteperonista.org: Frente Peronista

http://coalicioncivicaari.org.ar: Coalición Cívica - ARI

http://pro.com.ar: Propuesta Republicana

www.partidosocialista.com.ar: Partido Socialista

www.partidoencuentro.org.ar: Nuevo Encuentro

www.partidogen.com.ar: Generación para un Encuentro Nacional

www.proyecto-sur.com.ar: Movimiento Proyecto Sur

www.libresdelsur.org.ar: Libres del Sur

www.pdp.org.ar: Partido Demócrata Progresista

www.democratamza.com.ar/index.htm: Demócrata de Mendoza

www.unionportodos.org: Unión por Todos

\section{Paraguay}

www.anr.org.py Asociación Nacional Republicana Partido Colorado

www.plra.org.py: Partido Liberal Radical Auténtico

www.unace.org.py: Partido Unión Nacional de Ciudadanos Éticos

www.patriaquerida.org: Partido Patria Querida

www.pdp.org.py: Partido Demócrata Progresista

\section{Uruguay}

www.frenteamplio.org.uy: Frente Amplio

www.partidonacional.com.uy: Partido Nacional

www.partidocolorado.com.uy: Partido Colorado

www.partidoindependiente.org.uy: Partido Independiente

Recibido el 13 de diciembre de 2012.

Aceptado el 18 de noviembre de 2014. 